\title{
Line Source Representations for Shear Wave Birefringence Measurements in Transversely Isotropic Materials using Laser Ultrasonics
}

James B. Spicer* and Fan W. Zeng

Department of Materials Science and Engineering

The Johns Hopkins University

3400 North Charles Street

Baltimore, Maryland 21218

*Corresponding author: spicer@jhu.edu

\begin{abstract}
Line source representations for thermoelastic, laser excitation of transversely isotropic materials are developed to model shear wave birefringence effects that can occur in these materials. Continuum descriptions for elastic wave propagation are used to generate equations of motion that are solved using transform methods. Analytical solutions are presented for epicentral waveforms for propagation parallel and perpendicular to the axis of symmetry. These solutions are particularly simple and allow for rapid examination of the influence of various components of the stiffness tensor on wave propagation. In particular, for propagation perpendicular to the axis of symmetry, line source orientation parallel and perpendicular to this axis yield two cases that demonstrate shear wave birefringence. This effect is computed for titanium as well as for a nearly isotropic material - both Class I - to highlight the influence of diagonal as well as offdiagonal components of the stiffness tensor on the character of laser ultrasonic waveforms when the tensor is referenced to the principal coordinate system. The results presented in this work should be used to guide the interpretation of laser ultrasonic, line source measurements in transversely isotropic materials when birefringence effects are present.
\end{abstract}

Keywords: transverse isotropy, elastic wave, shear wave birefringence, laser ultrasonics, elasticity tensor

\subsection{Introduction}

Elastic waves derived from line sources have received the attention of researchers dating back to Lamb whose classic work on step loading of an elastic half-space has inspired innumerable, succeeding investigations including the results presented here [1-3]. In this work, we develop models for ultrasonic waves produced using laser line sources in transversely anisotropic materials with the aim of developing specific shear wave polarizations that can be used to make 
birefringence measurements and characterize aspects of the material anisotropy. These models will use idealized line source representations for the laser source to simplify the overall treatment and to highlight the effects of elastic anisotropy on wave propagation. Taking this approach allows for a concise presentation of analytical solutions and isolates specific characteristics of these solutions. It also captures the essential physical processes permitting easy computation for comparison to experimental measurements. These comparisons are not presented in this work, but various characteristics of wave propagation that have not been explored previously will be noted - these potentially point to new directions for materials characterization using elastic waves.

An important characteristic of materials that directly impacts elastic wave propagation is texture - the partial alignment of elastically anisotropic grains/crystallites. For a population of randomly-oriented grains that interact with ultrasound over sufficient propagation distances, materials can behave isotropically. However, if the grains do not have complete orientational randomization in the sampled volume, then the material will be elastically anisotropic and wavespeeds will vary with direction and polarization [4-6]. Beyond grain orientation effects, partial alignment of defect structures such as microcracks can also induce elastic anisotropy [710]. Ultrasound has been used to assess this anisotropy and, with appropriate models, can be used to infer some characteristics of the underlying microstructure such as the orientation distribution coefficients. In particular, shear wave birefringence measurements can be used to isolate specific aspects of the anisotropy that can be useful for process monitoring and control [11-13] and various methods have been implemented to make these types of measurements $[14,15]$, but none has taken advantage of laser line sources to produce polarized shear waves.

Laser line sources for elastic wave generation as well as the wavefields associated with these sources have been studied extensively. Early work simulated the behavior of thermoelastic sources using shear stress dipoles and focused on the directivity patterns associated with longitudinal and shear waves in isotropic materials [16]. Subsequent reports refined this general description by including details related to the underlying physics such as optical absorption, photothermal conversion and thermoelastic response [17-22]. Various approaches have been used to model characteristics of the resulting elastic wavefield and these have been verified experimentally [17]. One major finding is that the wave directivities and polarizations for these types of sources are complicated - even when the material is isotropic. Unlike other methods 
that have been used to perform ultrasonic shear birefringence measurements, the potential for using laser line sources for these types of measurements is largely unexplored. A good starting point for assessing this potential is to model shear wave birefringence in anisotropic materials excited using a laser source.

For isotropic materials, modeling of elastic waves generated by line sources has been the subject of many investigations. Indeed, Lamb's original problem is generally shown in textbooks on wave motion in elastic solids since it considers isotropic materials - the expressions for displacements in the wavefield are compact and readily interpreted [1,3,23]. The extension to anisotropic materials and point sources requires effort but yields rich results that go well beyond those to be exploited here [24-31]. Fortunately, line source excitation of transversely anisotropic half-spaces has also been considered in some detail and the essential mathematical techniques required to derive solutions is generally no more involved than those used for isotropic materials [32-34]. However, compact analytical solutions exist only for specific cases, but these can be obtained using careful algebraic manipulations. While direct numerical solution of the governing equations and boundary conditions can be performed [35,36], simple insights into the overall behavior of the system can be lost - analytical results permit general conclusions to be made that might be difficult to identify otherwise. The subsequent sections will develop models for the epicentral displacements resulting from laser line excitation of transversely isotropic halfspaces of different orientations. The development will largely follow approaches presented previously by Payton [37], Hurley [38] and others [39] with minor changes to notation to improve clarity. Three specific cases will be considered: Case I - Line source in the plane of symmetry, displacements along the symmetry axis; Case II - Line source perpendicular to the symmetry axis, displacements perpendicular to the symmetry axis; Case III - Line source parallel to the symmetry axis, displacements perpendicular to the symmetry axis. The geometry for each is shown in Fig. 1. While only Cases II and III are needed to illustrate shear wave birefringence, Case I shows the methods used for solution and complements the other results. In all cases, final solutions for epicentral displacements will be given for materials having convex normal surfaces (Class I materials) such as occurs for various single crystals with hexagonal symmetry [37] as well as for certain weakly-textured, polycrystalline materials displaying transverse isotropy.

\subsection{Theory and Models}


In the following developments, epicentral displacements from suitably-oriented, unit strength, shear stress dipoles will be derived. These stresses relate directly to the corresponding surface tractions that are appropriate for each case. The relationship of shear stress dipoles to laser line sources has been established previously and these are relatively simple for adiabatic conditions that ignore thermal diffusion [19,38,39]. Under these conditions, appropriate normalization factors can be applied to the results presented here to compute displacements.

\subsection{Epicentral Displacements Along Symmetry Axis - Case I}

Suppose we have a transversely isotropic material in the form of a half-space where the surface is perpendicular to the $\mathrm{x}_{3}$-axis. A shear stress is applied at the surface along the $x_{1}$-axis such that:

$$
\left.\sigma_{32}\right|_{x_{3}=0}=\delta^{\prime}\left(x_{2}\right) H(t)
$$

where $\sigma_{i j}$ is the elastic stress tensor, $\delta\left(x_{i}\right)$ is the Dirac delta (the prime indicates differentiation with respect to $x_{2}$ ) and $H(t)$ is the Heaviside step function where $t$ represents time. No normal tractions act at the boundary and this implies that the normal stress vanishes:

$$
\left.\sigma_{33}\right|_{x_{3}=0}=0 \text {. }
$$

These conditions mimic those for a laser line source in the thermoelastic (non-ablative) regime. The equation of motion for the medium can be written as:

$$
\sigma_{j i, j}+f_{i}=\rho u_{i, t t}
$$

where $f_{i}$ represents the component of the body force along the $x_{i}$ axis, $\rho$ is the material density and $u_{i}$ is the material displacement along the $x_{i}$ axis and indices after commas indicate differentiation - spatial coordinates are referenced using corresponding subscripts. The following constitutive relationship will be used:

$$
\sigma_{i j}=c_{i j k l} \varepsilon_{k l}
$$

where $c_{i j k l}$ are the components of the elastic stiffness tensor and $\varepsilon_{k l}$ is the infinitesimal strain tensor. This can be written in terms of the material displacement as follows:

$$
\varepsilon_{i j}=\left(u_{i, j}+u_{j, i}\right) / 2 \text {. }
$$

Using these relationships along with the equation of motion yields the following wave equation describing material displacements in the medium:

$$
c_{i j k l} u_{k, l j}+f_{i}=\rho u_{i, t t}
$$


where the symmetry of the stress tensor has been utilized. For transversely isotropic materials, the stiffness tensor exhibits a number of symmetries that can be used to reduce the number of independent, non-vanishing components to the following: $c_{11}=c_{22} \neq c_{33}, c_{12} \neq c_{13}=c_{23}, c_{44}=c_{55}$ and $c_{66}=\left(c_{11}-c_{12}\right) / 2$ where contracted notation has been used for the subscripting $(11 \rightarrow 1$, $22 \rightarrow 2,33 \rightarrow 3,23 \rightarrow 4,13 \rightarrow 5$ and $12 \rightarrow 6)$. Making the substitutions [37]:

$$
u_{i}=\left(\rho / c_{44}\right)^{1 / 2} v_{i} \quad \text { and } \quad t=\left(\rho / c_{44}\right)^{1 / 2} \tau
$$

in Eq. (6) yields the following:

$$
\left(c_{i j k l} / c_{44}\right) v_{k, l j}+f_{i}\left(\rho c_{44}\right)^{-1 / 2}=v_{i, \tau \tau}
$$

where the stiffness tensor components have been normalized by one of the shear stiffnesses and the body force is normalized by the corresponding elastic impedance. For the purposes of this presentation, it will be assumed that the laser source can be represented using boundary stresses alone and subsurface sources are negligible $\left(f_{i}=0\right)$. Considerations of the symmetry of the imposed boundary stresses indicate that $v_{1}=0$ and $v_{i, 1}=0$ - only displacements and derivatives associated with the $x_{2}$ and $x_{3}$ need to be considered. Using $\alpha=c_{33} / c_{44}, \beta=c_{11} / c_{44}$ and $\kappa=1+c_{23} / c_{44}$, Eq. (8) yields the following:

$$
\beta v_{2,22}+v_{2,33}+\kappa v_{3,23}=v_{2, \tau \tau}
$$

and

$$
\alpha v_{3,33}+v_{3,22}+\kappa v_{2,32}=v_{3, \tau \tau} .
$$

These equations need to be solved simultaneously and this will be accomplished using the Fourier-Laplace transform pair defined as follows:

$$
\bar{v}_{i}=\int_{0}^{\infty} \int_{-\infty}^{\infty} v_{i} \exp \left(-i k_{2} x_{2}-s \tau\right) d x_{2} d \tau
$$

and

$$
v_{i}=-i(2 \pi)^{-2} \int_{C} \int_{-\infty}^{\infty} \bar{v}_{i} \exp \left(i k_{2} x_{2}+s \tau\right) d x_{2} d s .
$$

Transforming Eqs. (9) and (10) and assuming $\bar{v}_{2}=A \exp \left(k_{3} x_{3}\right)$ along with $\bar{v}_{3}=B \exp \left(k_{3} x_{3}\right)$ yields two algebraic equations that must be solved simultaneously. The following characteristic equation results:

$$
\alpha k_{3}^{4}-k_{3}^{2}\left((1+\alpha) s^{2}+\chi k_{2}^{2}\right)+\left(\beta k_{2}^{4}+(1+\beta) k_{2}^{2} s^{2}+s^{4}\right)=0
$$


where $\chi=1+\alpha \beta-\kappa^{2}$. The form for this equation suggests that we can parameterize the propagation constants as follows: $k_{2}=s \gamma_{2}$ and $k_{3}=s \gamma_{3}$ [37]. The resulting equation is a quadratic for $\gamma_{3}^{2}$ (bi-quadratic for $\gamma_{3}$ ) and the resulting four roots are summarized as follows:

$$
\begin{aligned}
& \gamma_{3_{1}}=(2 \alpha)^{-1 / 2}\left\{\left[(1+\alpha)+\chi \gamma_{2}^{2}\right]+\varphi^{1 / 2}\right\}^{1 / 2} \\
& \gamma_{3_{2}}=-\gamma_{3_{1}} \\
& \gamma_{3_{3}}=(2 \alpha)^{-1 / 2}\left\{\left[(1+\alpha)+\chi \gamma_{2}^{2}\right]-\varphi^{1 / 2}\right\}^{1 / 2} \\
& \gamma_{3_{4}}=-\gamma_{3_{3}}
\end{aligned}
$$

where $\varphi=\left[(1+\alpha)+\chi \gamma_{2}{ }^{2}\right]^{2}-4 \alpha\left[\beta \gamma_{2}{ }^{4}+(1+\beta) \gamma_{2}{ }^{2}+1\right]$. Propagating solutions require that these roots be real and consideration of solution boundedness as $x_{3} \rightarrow \infty$ requires that only explicitly negative roots be retained in the full solution. The assumed forms for $\bar{v}_{2}$ and $\bar{v}_{3}$ are as follows:

$$
\begin{array}{r}
\bar{v}_{2}=a_{1} \exp \left(-s \gamma_{3_{1}} x_{3}\right)+a_{2} \exp \left(-s \gamma_{3_{3}} x_{3}\right) \\
\bar{v}_{3}=b_{1} \exp \left(-s \gamma_{3_{1}} x_{3}\right)+b_{2} \exp \left(-s \gamma_{3_{3}} x_{3}\right) .
\end{array}
$$

The four coefficients are determined using the transformed versions of Eqs. (8), (9), (2) and (1). In this work, we are only interested in displacements along the $x_{3}$ axis (since these can be readily measured) and will only present results for the coefficients $b_{1}$ and $b_{2}$. Clearly, direct computation of displacements can be carried out at this point, but there are advantages that can be gained by additional analytical manipulations of the various equations since relatively simple expressions for the displacements can be obtained ultimately. The extensive algebra involved is unwieldy and requires keen attention to groupings of terms, but in the end the following results are obtained:

$$
b_{1}=\left(c_{44} \rho\right)^{-1 / 2}\left(\kappa \gamma_{2}^{2} / s\right)\left[\left(1+\gamma_{2}^{2}\right)(1-\kappa)-\alpha \gamma_{3_{3}}^{2}\right] \gamma_{3_{1}} \div D
$$

and

$$
b_{2}=-\left(c_{44} \rho\right)^{-1 / 2}\left(\kappa \gamma_{2}^{2} / s\right)\left[\left(1+\gamma_{2}^{2}\right)(1-\kappa)-\alpha{\gamma_{3_{1}}}^{2}\right] \gamma_{3_{3}} \div D
$$

where

$$
D=\kappa\left(\gamma_{3_{1}}-\gamma_{3_{3}}\right)\left\{\left(1+\gamma_{2}^{2}\right)\left[2(1-\kappa) \gamma_{2}^{2}-\left(\chi \gamma_{2}^{2}+\alpha\right)\right]-\alpha \gamma_{3_{1}} \gamma_{3_{3}}\right\}
$$

Equations (16)-(19) provide the complete solution for the desired displacements in the doubly transformed domain. Fortunately, the process for performing the required inversions is well known. Even so, the various steps will be shown here for completeness. 
First, the inverse Fourier transform with respect to the variable $k_{2}=s \gamma_{2}$ will be performed:

$$
\tilde{v}_{3}=(2 \pi)^{-1} \int_{-\infty}^{\infty} \bar{v}_{3} \exp \left(i s \gamma_{2} x_{2}\right) s d \gamma_{2}=(\pi)^{-1} \operatorname{Re} \int_{0}^{\infty} \bar{v}_{3} \exp \left(i s \gamma_{2} x_{2}\right) s d \gamma_{2}
$$

where $\tilde{v}_{3}$ represents the Laplace transform of $v_{3}$ and the final equality in Eq. (20) results from $\bar{v}_{3}$ being even in $\gamma_{2}$.

Since only epicentral displacements are needed, $x_{2}=0$ and the transform simplifies considerably since the integrand in Eq. (20) depends on $\gamma_{2}{ }^{2}-$ this suggests a change in the integration variable. Letting $\Gamma_{2}=\gamma_{2}^{2}$ yields the following:

$$
\tilde{v}_{3}=(2 \pi)^{-1} \operatorname{Re} \int_{0}^{\infty} \bar{v}_{3} s \Gamma_{2}^{-1 / 2} d \Gamma_{2} .
$$

While this change might not appear to have achieved any significant advantage, it actually simplifies the remaining inverse transform operation - integration with respect to $\tau$ along $C$ allowing the ultimate solution to be obtained essentially by inspection. We will consider the terms containing $b_{1}$ and $b_{2}$ separately and begin with the $b_{1}$ term. For this term in the transform, let the variable $\tau_{1}=\gamma_{3_{1}} x_{3}$ stand in for $\tau$ in the inverse Laplace transform operation in Eq. (12). With this parameterization, $\Gamma_{2}$ can be interpreted to be a function of $\tau_{1}$ such that $d \Gamma_{2}=\frac{1}{x_{3}} \frac{\partial \Gamma_{2}}{\partial \gamma_{3_{1}}} d \tau_{1}$. A similar parameterization for the term containing $b_{2}$ can be carried out ( $\tau_{3}=\gamma_{3_{3}} x_{3}$ ) so that combining Eq. (21) with (20) (including (16)-(19)) yields the following:

$$
\begin{aligned}
v_{3}=-i(2 \pi)^{2}\left\{\int_{C} \operatorname{Re} \int_{0}^{\infty} H\left(\tau_{1}-x_{3}\right) b_{1} s \Gamma_{2}^{-1 / 2} \frac{\partial \Gamma_{2}}{\partial \tau_{1}} \exp \left(-s \tau_{1}\right) \exp (s \tau) d \tau_{1} d s\right. \\
\left.\quad+\int_{C} \operatorname{Re} \int_{0}^{\infty} H\left(\tau_{3}-\alpha^{-1 / 2} x_{3}\right) b_{2} s \Gamma_{2}^{-1 / 2} \frac{\partial \Gamma_{2}}{\partial \tau_{3}} \exp \left(-s \tau_{3}\right) \exp (s \tau) d \tau_{3} d s\right\}
\end{aligned}
$$

Equation (22) appears to be a forward Laplace transform immediately followed by the inverse Laplace transform operation. The result of these operations can be written directly as follows:

$$
v_{3}=(2 \pi)^{-1} \operatorname{Re}\left[H\left(\tau_{1}-x_{3}\right) b_{1} s \Gamma_{2}^{-1 / 2} \frac{\partial \Gamma_{2}}{\partial \tau_{1}}+H\left(\tau_{3}-\alpha^{-1 / 2} x_{3}\right) b_{2} s \Gamma_{2}^{-1 / 2} \frac{\partial \Gamma_{2}}{\partial \tau_{3}}\right]
$$

Next, we need to express $\Gamma_{2}$ in terms of $\gamma_{3}$ and also put the derivatives in Eq. (23) into recognizable forms. To achieve the first of these tasks, Eq. (13) can be written as a quadratic in $\Gamma_{2}$ and can be solved to yield the dependence of $\Gamma_{2}$ on $\gamma_{3}$ as follows: 


$$
\Gamma_{2}=\left.(2 \beta)^{-1}\left\{\chi \gamma_{3}^{2}-(1+\beta) \mathrm{m}\left[\left(\chi \gamma_{3}^{2}-(1+\beta)\right)^{2}-4 \beta\left(\alpha \gamma_{3}{ }^{4}-(1+\alpha) \gamma_{3}^{2}+1\right)\right]^{1 / 2}\right\}\right|_{\substack{\gamma_{3_{3}} \rightarrow-\\ \gamma_{3} \rightarrow+}}
$$

The derivative can be obtained most directly by differentiating the quadratric equation for $\Gamma_{2}$ and this yields the result:

$$
\frac{\partial \Gamma_{2}}{\partial \gamma_{3}}=\frac{2 \chi \gamma_{3} \Gamma_{2}+2(1+\alpha) \gamma_{3}-4 \alpha \gamma_{3}^{3}}{2 \beta \Gamma_{2}+(1+\beta)-\chi \gamma_{3}^{2}} .
$$

where the appropriate sign in Eq. (24) is chosen based on the term in Eq. (23) being evaluated. For completeness, this derivative is written for these terms using previous definitions as follows:

$$
\frac{\partial \Gamma_{2}}{\partial \tau_{1}}=\frac{1}{x_{3}} \frac{\partial \Gamma_{2}}{\partial \gamma_{3_{1}}}=\left.\frac{1}{x_{3}} \frac{\partial \Gamma_{2}}{\partial \gamma_{3}}\right|_{\gamma_{3}=\gamma_{31}} \text { and } \frac{\partial \Gamma_{2}}{\partial \tau_{3}}=\frac{1}{x_{3}} \frac{\partial \Gamma_{2}}{\partial \gamma_{3_{3}}}=\left.\frac{1}{x_{3}} \frac{\partial \Gamma_{2}}{\partial \gamma_{3}}\right|_{\gamma_{3}=\gamma_{3}} \text {. }
$$

With all terms expressed explicitly, it only remains to compute displacement values as a function of reduced time. For the first term in Eq. (23), we let $\tau=\tau_{1}=\gamma_{3_{1}} x_{3}$ and this is used to compute $\gamma_{3_{1}}$. This value for $\gamma_{3}$ is used in Eq. (24) to compute $\Gamma_{2}$ (choosing the correct sign) as well as the derivative in Eq. (26). This value for $\Gamma_{2}$ is used in (14c) to compute $\gamma_{3_{3}}$. Finally, these values for $\gamma_{3_{1}}, \gamma_{3_{3}}$ and $\Gamma_{2}$ are used with Eqs. (17) and (19) to evaluate the coefficient $b_{1}$. The second term is evaluated in a similar fashion but the parameterization begins with $\tau=\tau_{3}=\gamma_{3_{3}} x_{3}$. This completes the evaluation of displacements along the $x_{3}$ axis in a half space arising from a shear dipole line source with step time dependence acting on a surface perpendicular to the axis of symmetry in a transversely isotropic material. These displacements are independent of the orientation of the line on this surface since it is a plane of isotropy. The strength of the laser line source dipole can be obtained by multiplying the unit source used here by $q_{0}\left[\beta_{22}-\left(c_{23} / c_{33}\right) \beta_{33}\right] / C$ where $q_{0}$ is the line fluence $[\mathrm{J} / \mathrm{m}], \beta_{i j}$ are the components of the thermal stress tensor and $C$ is the heat capacity $\left[\mathrm{Jm}^{-3} \mathrm{~K}^{-1}\right][38,39]$.

\subsection{Epicentral Displacements Perpendicular to Symmetry Axis: Line Source Perpendicular to} Symmetry Axis - Case II

For this case, the surface of the half-space is perpendicular to the $x_{2}$-axis and a shear stress is applied on the surface along the $x_{1}$-axis such that:

$$
\left.\sigma_{23}\right|_{x_{2}=0}=\delta^{\prime}\left(x_{3}\right) H(t)
$$


and it is assumed that there are no contributions from the normal stress to the surface tractions such that:

$$
\left.\sigma_{22}\right|_{x_{2}=0}=0
$$

Owing to the symmetries of these stresses, once again, only displacements and derivatives associated with the $x_{2}$ and $x_{3}$ directions need to be considered. The equations of motion, given in Eqs. (9) and (10), are unchanged and the overall solution process follows the previous development. Indeed, since we are interested in epicentral displacements $\left(x_{1}=0\right)$, it can be used in its entirety if the indices are exchanged ( $2 \bar{A} 3$ ) and the definitions of the normalized moduli are updated $\left(\alpha=c_{22} / c_{44}, \beta=c_{33} / c_{44}\right)$.

\subsection{Epicentral Displacements Perpendicular to Symmetry Axis: Line Source Parallel to} Symmetry Axis - Case III

For this case, the surface of the half-space is perpendicular to the $x_{2}$-axis and a shear stress is applied on the surface containing the $x_{3}$-axis such that:

$$
\left.\sigma_{21}\right|_{x_{2}=0}=\delta^{\prime}\left(x_{1}\right) H(t)
$$

and it is assumed that there are no contributions from the normal stress to the surface tractions such that:

$$
\left.\sigma_{22}\right|_{x_{2}=0}=0
$$

Owing to the symmetries of these stresses, only displacements and derivatives associated with the $x_{1}$ and $x_{2}$ directions need to be considered. Since we are interested in epicentral displacements $\left(x_{3}=0\right)$, it appears that the previous development can be used in its entirety with a change of indices $(3 \rightarrow 1)$. However, with this change the reduced time and displacements would need to be redefined along with the normalized moduli (the shear wave travels at a different speed from the previous cases). Since the previous solutions were expressed in terms of these quantities, the treatment here will maintain the definitions for reduced time and displacement. With the following normalized modulus definitions: $\alpha=c_{11} / c_{44}=c_{22} / c_{44}$, $\eta=c_{66} / c_{44}$ and $\kappa=\eta+c_{12} / c_{44}$, the equations of motion are expressed as follows:

$$
\alpha v_{1.11}+\eta v_{1,22}+\kappa v_{2,12}=v_{1, \tau \tau}
$$

and 


$$
\alpha v_{2,22}+\eta v_{2,11}+\kappa v_{1,21}=v_{2, \tau \tau} .
$$

Recalling that $c_{66}=\left(c_{11}-c_{12}\right) / 2$, the normalized moduli are related $(\kappa=\alpha-\eta)$ and only two independent moduli remain. This indicates the material behaves isotropically for the conditions imposed here since the elastic response of isotropic materials can be described using two moduli. The solution to this problem is well known and can be written directly. Even so, we will choose to follow the solution process outlined previously, and compare the result to ones already known. Using notation developed previously, the following expression for the epicentral displacement is obtained:

$$
v_{2}=(2 \pi)^{-1} \operatorname{Re}\left[H\left(\tau_{1}-\eta^{-1 / 2} x_{2}\right) b_{1} s \Gamma_{1}^{-1 / 2} \frac{\partial \Gamma_{1}}{\partial \tau_{2}}+H\left(\tau_{3}-\alpha^{-1 / 2} x_{2}\right) b_{2} s \Gamma_{1}^{-1 / 2} \frac{\partial \Gamma_{1}}{\partial \tau_{3}}\right]
$$

where $\tau_{1}=\gamma_{2_{1}} x_{2}, \tau_{3}=\gamma_{2_{3}} x_{2}$ and

$$
\begin{aligned}
& \gamma_{2_{1}}=(2 \alpha \eta)^{-1 / 2}\left\{\left[(\alpha+\eta)+\chi \gamma_{1}^{2}\right]+\varphi^{1 / 2}\right\}^{1 / 2}=\left(\gamma_{1}^{2}+\eta^{-1}\right)^{1 / 2} \\
& \gamma_{2_{3}}=(2 \alpha \eta)^{-1 / 2}\left\{\left[(\alpha+\eta)+\chi \gamma_{1}^{2}\right]-\varphi^{1 / 2}\right\}^{1 / 2}=\left(\gamma_{1}{ }^{2}+\alpha^{-1}\right)^{1 / 2}
\end{aligned}
$$

with $\chi=\alpha^{2}+\eta^{2}-\kappa^{2}=2 \alpha \eta$ and $\varphi=\left[(\alpha+\eta)+\chi \gamma_{1}^{2}\right]^{2}-4 \alpha \eta\left(\alpha \gamma_{1}^{2}+1\right)\left(\eta \gamma_{1}^{2}+1\right)=\eta-\alpha$. The coefficients, $b_{1}$ and $b_{2}$, for this case are:

$$
\begin{aligned}
b_{1} & =-\left(c_{44} \rho\right)^{-1 / 2}\left(\kappa \gamma_{1}^{2} /(\eta s)\right)\left[(\eta-\kappa)\left(1+\eta \gamma_{1}^{2}\right)-\alpha \eta \gamma_{2_{3}}{ }^{2}\right] \gamma_{2_{1}} \div D \\
& =\left(c_{44} \rho\right)^{-1 / 2}\left(\kappa^{2} \gamma_{1}^{2} \gamma_{2_{1}}\right)\left(1+2 \eta \gamma_{1}^{2}\right) /(\eta s) \div D
\end{aligned}
$$

and

$$
\begin{aligned}
b_{2} & =\left(c_{44} \rho\right)^{-1 / 2}\left(\kappa \gamma_{1}{ }^{2} /(\eta s)\right)\left[(\eta-\kappa)\left(1+\eta \gamma_{1}{ }^{2}\right)-\alpha \eta \gamma_{2_{1}}{ }^{2}\right] \gamma_{2_{3}} \div D \\
& =-\left(c_{44} \rho\right)^{-1 / 2}\left(2 \kappa^{2} \gamma_{1}{ }^{2} \gamma_{2_{1}}{ }^{2} \gamma_{2_{3}} / s\right) \div D
\end{aligned}
$$

with

$$
\begin{aligned}
D & \left.=\kappa\left(\gamma_{2_{1}}-\gamma_{2_{3}}\right)\left\{\left(1+\eta \gamma_{1}^{2}\right)\left[(2(\kappa-\eta)+\chi / \eta) \gamma_{1}^{2}+\alpha / \eta\right)\right]+\alpha \gamma_{2_{1}} \gamma_{2_{3}}\right\} \\
& =\kappa^{2} \eta \gamma_{2_{1}}\left[\left(2 \gamma_{1}^{2}+1 / \eta\right)^{2}-4 \gamma_{1}^{2} \gamma_{2_{1}} \gamma_{2_{3}}\right]
\end{aligned}
$$

where the second expression for $D$ is readily recognized as being related to the Rayleigh denominator [23]. As expected, the coefficients $b_{1}$ and $b_{2}$ agree with previous developments for isotropic materials $[17,19]$. Using this form for the transformed solution, the inversions can be carried out. First, $\Gamma_{1}=\gamma_{1}^{2}$ must be expressed in terms of $\gamma_{2}$ and the derivatives in Eq. (31) must be written in a recognizable form. Following the steps presented for the first case yields: 


$$
\Gamma_{1}=\left.(2 \alpha \eta)^{-1}\left\{\chi \gamma_{2}^{2}-(\alpha+\eta) \mathrm{m}\left[\left(\chi \gamma_{2}^{2}-(\alpha+\eta)\right)^{2}-4 \alpha \eta\left(\alpha \gamma_{2}^{2}-1\right)\left(\eta \gamma_{2}^{2}-1\right)\right]^{1 / 2}\right\}\right|_{\substack{\gamma_{2} \rightarrow-\\ \gamma_{23} \rightarrow+}},(38)
$$

where the appropriate sign in Eq. (38) is chosen based on the term in Eq. (33) being evaluated. For this case, the results for $\Gamma_{1}$ are simple - both Eq. (38) and (34) give $\Gamma_{1}=\gamma_{2_{1}}^{2}-\eta^{-1}$ or $\Gamma_{1}=\gamma_{2_{3}}^{2}-\alpha^{-1}$. The corresponding derivative

$$
\frac{\partial \Gamma_{1}}{\partial \gamma_{2}}=\frac{2 \chi \gamma_{2} \Gamma_{1}+2(\alpha+\eta) \gamma_{2}-4 \alpha \eta \gamma_{2}^{3}}{2 \alpha \eta \Gamma_{1}+(\alpha+\eta)-\chi \gamma_{2}^{2}}=2 \gamma_{2}
$$

simplifies considerably as well. For completeness, this derivative is written for various terms using previous definitions as follows:

$$
\frac{\partial \Gamma_{1}}{\partial \tau_{1}}=\frac{1}{x_{2}} \frac{\partial \Gamma_{1}}{\partial \gamma_{2_{1}}}=\left.\frac{1}{x_{2}} \frac{\partial \Gamma_{1}}{\partial \gamma_{2}}\right|_{\gamma_{2}=\gamma_{2_{1}}}=\frac{2 \gamma_{2_{1}}}{x_{2}} \quad \frac{\partial \Gamma_{1}}{\partial \tau_{3}}=\frac{1}{x_{2}} \frac{\partial \Gamma_{1}}{\partial \gamma_{2_{3}}}=\left.\frac{1}{x_{2}} \frac{\partial \Gamma_{1}}{\partial \gamma_{2}}\right|_{\gamma_{2}=\gamma_{23}}=\frac{2 \gamma_{2_{3}}}{x_{2}}
$$

To compute displacements, we let $\tau=\tau_{1}=\gamma_{2_{1}} x_{2}$ (this defines a value for $\gamma_{2_{1}}$ ). This value is used to compute $\Gamma_{1}$ as well as the derivative in Eq. (40). The result for $\Gamma_{1}$ along with Eq. (34b) yield $\gamma_{2_{3}}$. Finally, these values for $\gamma_{2_{1}}, \gamma_{2_{3}}$ and $\Gamma_{1}$ are used with Eqs. (35) and (37) to evaluate the coefficient $b_{1}$. The second term is evaluated in a similar fashion but the parameterization begins with $\tau=\tau_{3}=\gamma_{2_{3}} x_{2}$. This completes the evaluation of displacements along the $x_{2}$ axis in a half space arising from a line source parallel to the axis of symmetry with step time dependence acting on a surface containing the axis of symmetry. The strength of the laser line source dipole for this case can be obtained by multiplying the unit source by $q_{0} \alpha_{L} c_{11}^{-1}\left(c_{11}+2 c_{12}\right)\left(c_{11}-c_{12}\right) / C$ where $\alpha_{L}$ is the linear thermal expansion coefficient - other terms were defined previously [38,39]. This result agrees with previous developments for laser line source excitation of isotropic half-spaces [19].

\subsection{Results - Evaluation of Displacements}

The reduced displacements expressed in Eqs. (23) and (31) will be evaluated for the epicentral range $x_{i}=1$ as a function of the reduced time, $\tau$, since this simplifies interpretation of the results. Also, only anisotropy that conforms to the requirements of Class I systems will be considered [40] - namely, those for which $\alpha>\beta$ and $\alpha+1<\chi<\chi_{0}(\alpha, \beta)$ where $\chi_{0}$ is a positive root of the cubic equation:

$$
0=\chi^{3}+l \chi^{2}+h \chi+n
$$


with

$$
\begin{aligned}
& l=1+\alpha \beta+2(1-\alpha)(1-\beta), \\
& h=-(\alpha+\beta)[(1-\alpha)+(1-\beta)+2 \alpha \beta], \\
& n=-\left[(\alpha+\beta)^{2}(1+\alpha \beta)+16 \alpha \beta(1-\alpha)(1-\beta)\right] .
\end{aligned}
$$

While other systems adhering to the requirements of Classes II-V could be considered [37], the solution processes required for these systems demand additional attention to the computation of inverse transforms. Related details can be found in work by Payton [37] and Hurley [38]. In addition, the solutions generated here for transversely isotropic materials might also apply (with restrictions) to materials displaying certain tetragonal (422, $4 \mathrm{~mm}, \overline{4} 2 \mathrm{~m}, 4 / \mathrm{mmm})$ and cubic symmetries when appropriate definitions for directions and modulus components are made. Since the focus of the current presentation is on transversely isotropic materials, displacements will be computed for titanium which, in single crystal form, adheres to the Class I requirements and has the following normalized moduli: $\alpha=c_{33} / c_{44}=3.88, \quad \beta=c_{11} / c_{44}=3.47$, $c_{23} / c_{44}=1.48$ and $c_{12} / c_{44}=1.97 \quad\left(c_{44}=46.7 \mathrm{GPa}\right)[37,40]$. Using these values, epicentral displacements were computed using the previous development for the three cases and are shown in Fig. 2. For Case I, the orientation of the line source has no effect on the arrival times of the either the shear or longitudinal waves since the plane of isotropy is perpendicular to the propagation direction and shear waves are polarized in this plane. For Cases II and III the longitudinal waves propagate at the same speed since the direction of propagation is the same, but the respective shear waves arrive at different times - these are the cases that display shear wave birefringence. Beyond the relative wavespeeds for the different modes in Cases II and III, the general shapes of the waveforms between the wave arrivals differ significantly. These shapes are dictated by the form of the solution related to the longitudinal wave - the wavespeed depends on $\beta$ while the overall shape depends on various factors that include $c_{23}$ in Case II and $c_{12}$ in Case III. This result is significant since it illustrates that the shape of waveforms can be used to infer principal components of the modulus tensor that do not affect wavespeed when propagation along principal directions is considered.

Even though titanium illustrates the necessary results related to shear birefringence, a second material is considered in which the anisotropy is not nearly as strong. While this material is 
hypothetical, it is meant to represent materials that might otherwise be isotropic (such as polycrystalline materials with random grain orientation) but have an induced anisotropy that results from some type of forming operation (such as preferred grain orientation that occurs in metallic materials during drawing/extrusion) or externally applied, uniaxial stress (that causes changes to the stiffnesses according to the third order elastic constants) [41]. For this case, the following values for the various moduli have been assumed: $\alpha=c_{33} / c_{44}=4.00$, $\beta=c_{11} / c_{44}=3.60, c_{23} / c_{44}=1.79$ and $c_{12} / c_{44}=1.80-$ these values yield $c_{66} / c_{44}=0.90$. This material is also Class I and adheres to the same restrictions on moduli as titanium. Results for the epicentral displacements are shown in Fig. 3. The overall trends for wavespeed are the same as for titanium - differences exist between Cases I and II/III for the longitudinal wavespeeds; Cases II and III display shear birefringence. Even so, since the assumed values for $c_{23}$ and $c_{12}$ are much closer in this material, the waveform shapes for Cases I-III are relatively similar when compared to those obtained for titanium.

\subsection{Discussion}

The models developed here can be used for the interpretation of laser ultrasonic, line source measurements in transversely isotropic materials - specifically those that satisfy Class I restrictions. To facilitate these types of measurements, some knowledge of the symmetry axis direction can be used to guide choices regarding propagation direction and polarization orientation. Absent orientation information, symmetry axis direction can be determined by screening for shear wave birefringence - so long as the sample geometry allows for measurements along and perpendicular to the symmetry axis. For propagation perpendicular to the symmetry axis, the results presented here indicate that only two measurements are required to accurately characterize birefringence. However, in practice, multiple line orientations might be necessary to accurately identify maximum and minimum shear wavespeeds especially if the direction of the symmetry axis is not clearly defined. This uncertainty in axis direction can arise in systems where applied stresses induce anisotropy in materials that might otherwise behave isotropically. For these types of measurements, the laser line source will generally launch shear waves that propagate at wavespeeds characteristic of the normal modes of the system and the quantity of energy deposited into each mode will depend on the orientation of the line relative to the symmetry axis. While the case of the arbitrarily-oriented line source has not been modeled here, it can be assumed that the measured waveforms would yield shear wavespeeds intermediate 
between the extrema presented by Cases II and III. These intermediate values might not accurately reflect the actual constitution of the material and could simply represent an artifact of the measurement process.

Unlike the idealized conditions represented in the models developed here, actual measurements can be complicated by factors that reflect the characteristics of the material. For example, in Cases II and III, the longitudinal wave time-of-arrival should be independent of line orientation and this is likely the case even if the laser line is not uniformly intense across the sample surface. However, if there are variations in the photothermal/thermoelastic conversion processes related to sample inhomogeneity as the line orientation is varied, then the arrival time for the longitudinal wave will change - this same inhomogeneity will affect shear wave times-of-arrival and could interfere with accurate identification of birefringence associated with elastic anisotropy. Also, microstructural variations that result in elastic inhomogeneity could produce variations in wave arrivals that might impact shear birefringence measurements. However, if the times-of-arrival for the longitudinal wave do not vary with line orientation, then this could be a good indication that any variation in shear wave arrival times is a result of anisotropy and not inhomogeneity.

For ultrasonic characterization methods, wavespeeds have been historically used to characterize elastic anisotropy since they relate directly to the stiffnesses of the material. Shear wave birefringence, as determined by wavespeed differences between orthogonally polarized modes, is one effect that can occur and this has been the focus of the presentation here. However, material anisotropy yields effects beyond those typically associated with birefringence. In particular, the character of displacements after the longitudinal arrival depends on components of the tensor that do not influence wavespeeds in bulk media for propagation along high symmetry directions. These displacements occur when finite sources act and are related to off-diagonal components of the stiffness tensor $-c_{2233}$ and $c_{1122}$ for the cases considered in this work. These components factor into the Poisson effect and can be measured using various techniques. For isotropic materials, they can be determined using measurements of longitudinal and shear wavespeeds. For transversely anisotropic materials, additional measurements are required. Longitudinal wavespeed in a long, thin rod depends on the anisotropic Young's modulus - this along with bulk wavespeeds can be used to determine the values of off-diagonal tensor components. The 
results presented here offer the possibility of determining values for three tensor components from a single waveform obtained using a single experiment. This has not been investigated previously, but offers paths for investigation that could resolve long-standing issues related to point- and line-source Green's function interpretation of elastic wave measurements. Ultimately, simple and robust methods for determining off-diagonal components of the stiffness tensor might be used to infer characteristics of materials microstructure that are not captured when isotropic material behavior is assumed.

\subsection{Conclusions}

Models for ultrasonic waves produced using laser line sources in transversely anisotropic materials have been presented that show how shear wave birefringence measurements using laser sources can be used to characterize aspects of material anisotropy. By assuming adiabatic heating as well as uniform line intensity and homogeneous photothermal conversion processes, these models provide a simple description that highlights the effects of elastic anisotropy on wave propagation. The analytical solutions in this work allow for easy computation for comparison to experimental measurements and also permit specific characteristics of waveforms to be related to various stiffness components. For propagation in high symmetry directions, diagonal components of the elastic stiffness tensor dictate the wavespeeds of longitudinal and shear waves. While these arrivals are notable and are the focus of most ultrasonics measurements, the results in this study show how off-diagonal components can affect the overall shape of laser ultrasonic waveforms between wavefronts - the possibility exists to extract three stiffness tensor components from a single waveform. Since most materials with crystalline microstructural elements display some level of elastic anisotropy, laser line source measurements of the kind outlined here could provide insight into the constitution of the microstructure with a limited number of measurements.

\section{Acknowledgements}

The authors gratefully acknowledge the support of the U.S. Department of Energy (DOE) through the Nuclear Energy University Program (NEUP) Contract No. 00118687. 


\section{References}

[1] H Lamb. On the propagation of tremors over the surface of an elastic solid, Philosophical Transactions of the Royal Society of London Series a-Containing Papers of a Mathematical or Physical Character. 203 (1904) 1-42.

[2] SK Bhandari, R Chander. On the exact evaluation of displacements within a semi-infinite solid due to a buried line source, Proceedings of the Indian Academy of Sciences-Earth and Planetary Sciences. 93 (1984) 37-45.

[3] E Kausel. Lamb's problem at its simplest, Proceedings of the Royal Society A-Mathematical Physical and Engineering Sciences. 469 (2013).

[4] M Hirao, N Hara, H Fukuoka, K Fujisawa. Ultrasonic monitoring of texture in cold-rolled steel sheets, J.Acoust.Soc.Am. 84 (1988) 667-672.

[5] F Reverdy, B Audoin. Elastic constants determination of anisotropic materials from phase velocities of acoustic waves generated and detected by lasers, J.Acoust.Soc.Am. 109 (2001) 1965-1972.

[6] S Dixon, C Edwards, SB Palmer. Texture measurements of metal sheets using wideband electromagnetic acoustic transducers, Journal of Physics D-Applied Physics. 35 (2002) 816824.

[7] S Crampin, S Peacock. A review of shear-wave splitting in the compliant crack-critical anisotropic Earth, Wave Motion. 41 (2005) 59-77.

[8] D Su, MH Santare, GA Gazonas. The effect of crack face contact on the anisotropic effective moduli of microcrack damaged media, Eng.Fract.Mech. 74 (2008) 1436-1455.

[9] D Su, MH Santare, GA Gazonas. An effective medium model for elastic waves in microcrack damaged media, Eng.Fract.Mech. 75 (2008) 4104-4116.

[10] X Wang, MH Santare, GA Gazonas. Anisotropic effective moduli of microcracked materials under antiplane loading, Eng.Fract.Mech. 76 (2009) 1910-1919.

[11] R Mloszewski, DA Hutchins, J Francoeur. Characterization of roll-drawn polypropylene using shear-wave birefringence, J.Mater.Sci. 27 (1992) 2413-2419.

[12] E Schneider. Ultrasonic birefringence effect - its application for materials characterizations, Optics and Lasers in Engineering. 22 (1995) 305-323. 
[13] A Moreau, D Levesque, M Lord, M Dubois, JP Monchalin, C Padioleau, et al. On-line measurement of texture, thickness and plastic strain ratio using laser-ultrasound resonance spectroscopy, Ultrasonics. 40 (2002) 1047-1056.

[14] B Dutton, RJ Dewhurst. Anisotropy measurements in metal alloys using a laser/electromagnetic acoustic transducer array system, Appl.Phys.Lett. 89 (2006).

[15] S Dixon, MP Fletcher, G Rowlands. The accuracy of acoustic birefringence shear wave measurements in sheet metal, J.Appl.Phys. 104 (2008).

[16] DA Hutchins, RJ Dewhurst, SB Palmer. Directivity patterns of laser-generated ultrasound in aluminum, J.Acoust.Soc.Am. 70 (1981) 1362-1369.

[17] JR Bernstein, JB Spicer. Line source representation for laser-generated ultrasound in aluminum, J.Acoust.Soc.Am. 107 (2000) 1352-1357.

[18] D Royer. Mixed matrix formulation for the analysis of laser-generated acoustic waves by a thermoelastic line source, Ultrasonics. 39 (2001) 345-354.

[19] I Arias, JD Achenbach. Thermoelastic generation of ultrasound by line-focused laser irradiation, Int.J.Solids Structures. 40 (2003) 6917-6935.

[20] JD Achenbach. Laser excitation of surface wave motion, J.Mech.Phys.Solids. 51 (2003) 1885-1902.

[21] I Arias, JD Achenbach. A model for the ultrasonic detection of surface-breaking cracks by the scanning laser source technique, Wave Motion. 39 (2004) 61-75.

[22] WX Hu, ML Qian. Acoustic field excited by a pulsed laser line source in a cylinder, Ultrasonics. 44 (2006) E1187-E1190.

[23] KF Graff. Wave Motion in Elastic Solids, Dover Publications, New York, 1991.

[24] RG Payton. 2-dimensional pulse propagation in a 2-parameter anisotropic elastic solid, Q Appl Math. 27 (1969) 147-\&.

[25] RG Payton. 2-dimensional anisotropic elastic waves emanating from a point source, Proceedings of the Cambridge Philosophical Society-Mathematical and Physical Sciences. 70 (1971) 191-\&.

[26] RL Ryan. Pulse propagation in a transversely isotropic half-space, J.Sound Vibrat. 14 (1971) $511-\&$.

[27] RG Payton. Epicenter motion of a transversely isotropic elastic half-space due to a suddenly applied buried point source, Int.J.Eng.Sci. 17 (1979) 879-887. 
[28] MJP Musgrave, RG Payton. Head wave contributions to elastic wave fields in a transversely isotropic half-space, Q J Mech Appl Math. 34 (1981) 235-250.

[29] RG Payton. Epicenter and epicentral-axis motion of a transversely isotropic elastic halfspace, SIAM J Appl Math. 40 (1981) 373-389.

[30] RL Weaver, W Sachse, KY Kim. Transient elastic waves in a transversely isotropic plate, Journal of Applied Mechanics-Transactions of the ASME. 63 (1996) 337-346.

[31] A Rahman, F Ahmad. Representation of the displacement in terms of scalar functions for use in transversely isotropic materials, J.Acoust.Soc.Am. 104 (1998) 3675-3676.

[32] EA Kraut. Advances in the theory of anisotropic elastic wave propagation, Rev.Geophys. 1 (1963) 401-448.

[33] RG Payton. Symmetry-axis elastic-waves for transversely isotropic media, Q Appl Math. 35 (1977) 63-73.

[34] RG Payton. Plane strain displacement and stress waves induced in a transversely isotropic elastic solid by a line source, Zeitschrift Fur Angewandte Mathematik Und Physik. 29 (1978) 262-272.

[35] JJ Wang, BQ Xu, ZH Shen, XW Ni, J Lu. Numerical simulation of thermoelastic stress field and laser ultrasound in transversely isotropic plate, Japanese Journal of Applied Physics. 47 (2008) 956-963.

[36] Y Pan, M Perton, C Rossignol, B Audoin. The transient response of a transversely isotropic cylinder under a laser point source impact, Ultrasonics. 44 (2006) E823-E827.

[37] RG Payton. Elastic Wave Propagation in Transversely Isotropic Media, Martinus Nijhoff, The Hague, Netherlands, 1983.

[38] DH Hurley. An Investigation of the Anisotropic and Heterogeneous Nature of Laser Generated Ultrasound in Carbon-Fiber-Reinforced Epoxy and Single Crystal Materials, The Johns Hopkins University, Baltimore, Maryland, 1997.

[39] DH Hurley, JB Spicer. Line source representation for laser-generated ultrasound in an elastic transversely isotropic half-space, J.Acoust.Soc.Am. 116 (2004) 2914-2922.

[40] MJP Musgrave, RG Payton. Criteria for elastic-waves in anisotropic media - a consolidation, Journal of Elasticity. 14 (1984) 269-285. 
[41] CM Sayers, DR Allen. The influence of stress on the principal polarization directions of ultrasonic shear-waves in textured steel plates, Journal of Physics D-Applied Physics. 17 (1984) 1399-1413. 


\section{Figure Captions:}

Figure 1: Geometries for laser line source excitation of a transversely isotropic half-space. The three cases here illustrate the relationship of the surface- and source-orientation relative to the symmetry axis and also show the epicentral locations where displacements are evaluated.

Figure 2: Epicentral waveforms for laser-line excitation of single-crystal titanium half-spaces corresponding to the three geometries illustrated in Fig. 1. Waveforms are shown offset for clarity.

Figure 3: Epicentral waveforms for laser-line excitation of transversely-isotropic half-spaces corresponding to the three geometries illustrated in Fig. 1. The moduli for this material represent those that might result from preferred grain or microcrack orientations that yield transverse isotropy. Waveforms are shown offset for clarity. 
Figure 1:

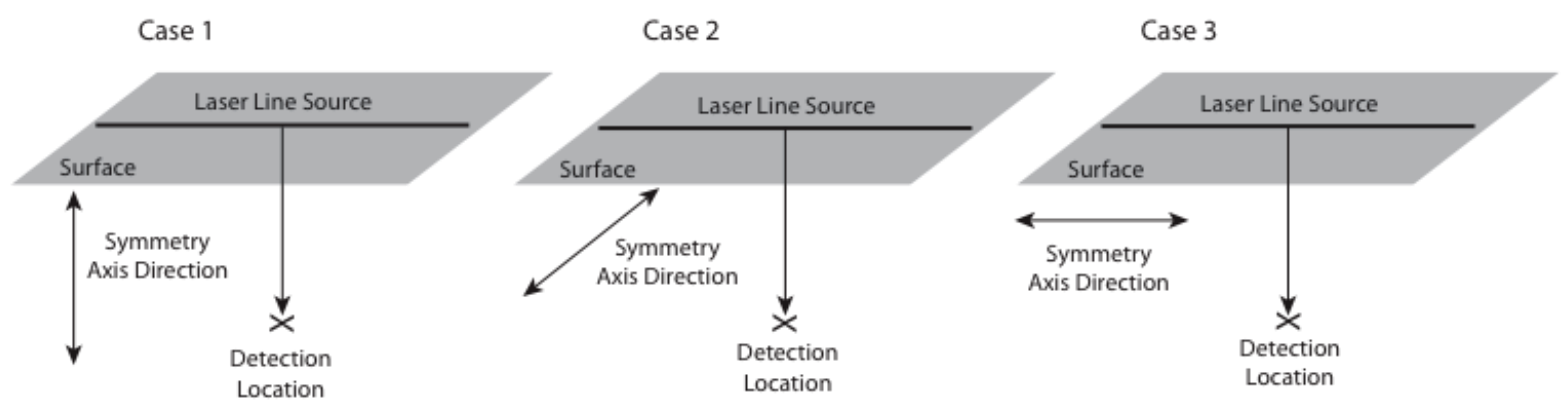


Figure 2:

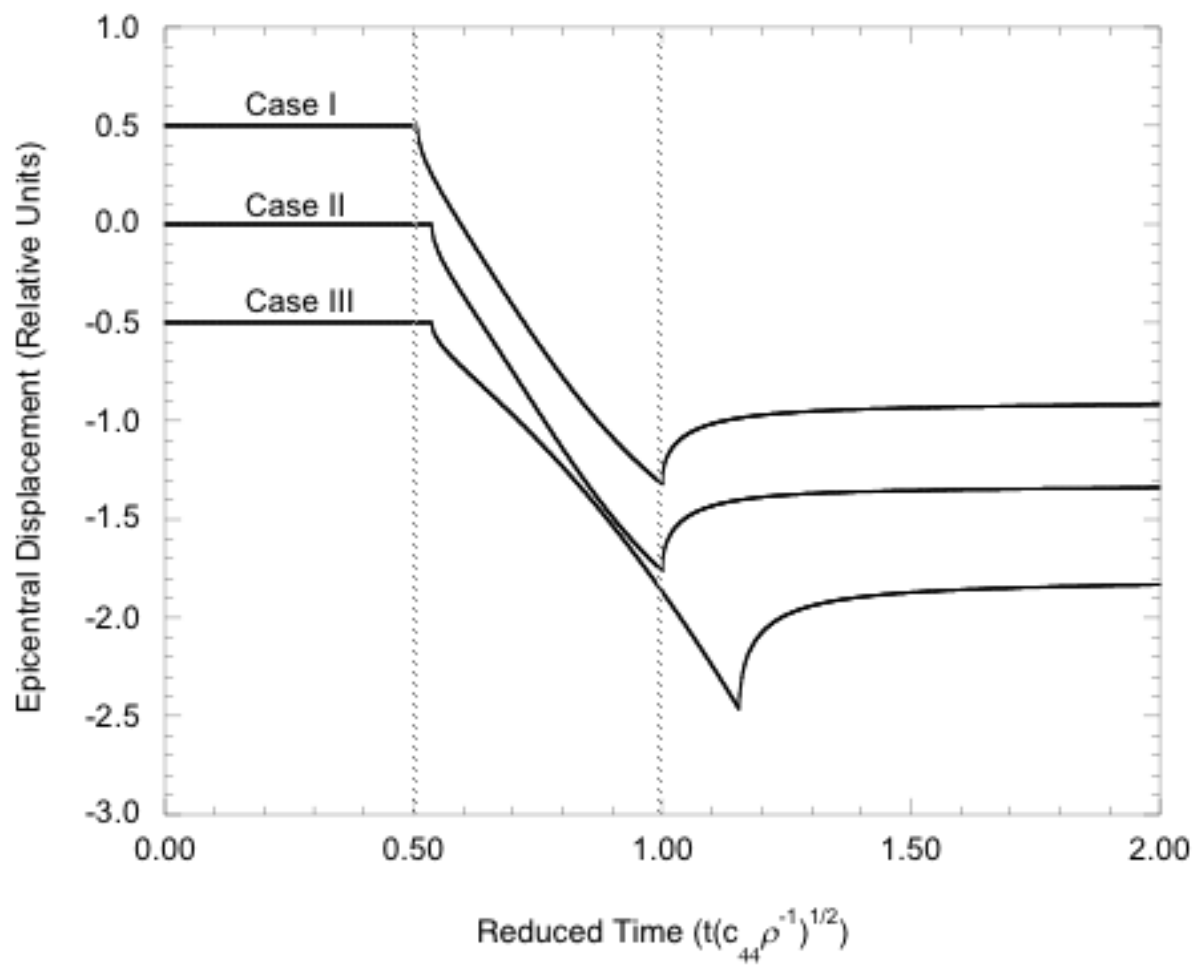


Figure 3:

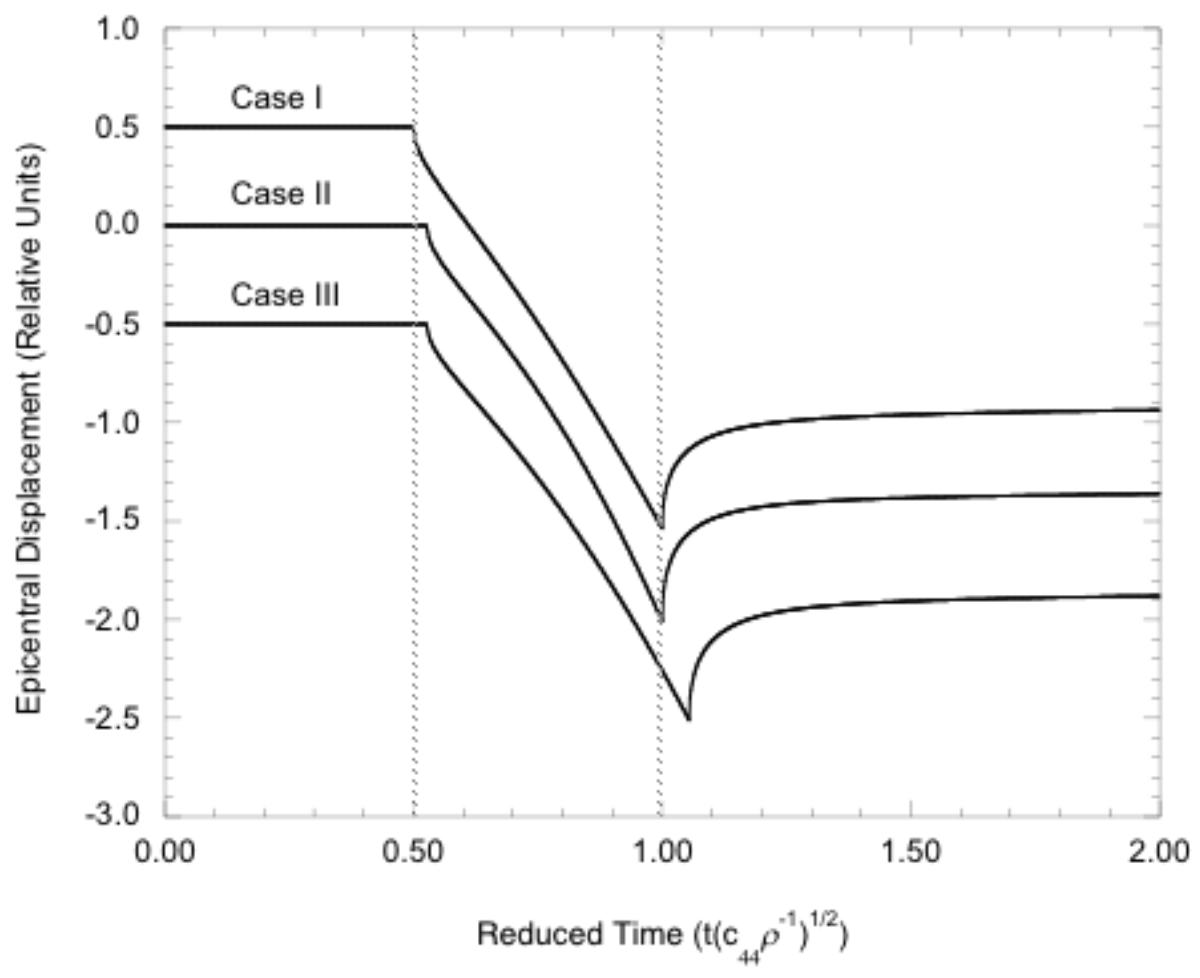

